\title{
Fire Needle Acupuncture Treatment for Lateral Epicondylitis (Tennis Elbow)
}

\author{
Jihe Zhu ${ }^{1}$, Blagica Arsovska², Kristina Kozovska ${ }^{1, *}$ \\ ${ }^{1}$ Faculty of Medical Sciences, University GoceDelcev, Shtip, Republic of Macedonia \\ ${ }^{2}$ Institute of Biology, Faculty of Natural Sciences and Mathematics, Skopje, Republic of Macedonia
}

\section{Email address:}

tcm.tongda.mk@gmail.com (Jihe Zhu),blagica25@gmail.com (B. Arsovska), tongdatang-tcm@hotmail.com (K. Kozovska)

*Corresponding author

\section{To cite this article:}

Jihe Zhu, Blagica Arsovska, Kristina Kozovska. Fire Needle Acupuncture Treatment for Lateral Epicondylitis (Tennis Elbow). American Journal of Clinical and Experimental Medicine. Vol. 5, No. 3, 2017, pp. 60-63. doi: 10.11648/j.ajcem.20170503.11

Received: February 1, 2017; Accepted: March 4, 2017; Published: March 22, 2017

\begin{abstract}
Lateral epicondylitis i.e. tennis elbow is a medical term for inflammation of the elbow on the lateral (outer) epicondyle. The condition usually occurs during excessive use of the arm, forearm and the muscles in that area which result with pain in the elbow. Fundamentally the condition appears when there are strong, monotonous and long movements of the muscles which burden the lateral epicondyle in the places of their attachment. When treating the lateral epicondylitits with Traditional Chinese Medicine (TCM), the first choice for a treatment is acupuncture. In our study are included 12 patients, 9 male and 3 female, all with symptoms of lateral epicondylitis and all treated with fire needle acupuncture. The treatments were done in a clinic for TCM and acupuncture in Skopje, Macedonia, by a doctor specialist in acupuncture. In the treatment were treated trigger i.e. Ashi points. Patients were on age from 24 to 70, with most common age group of 30-40. Number of the treatments is different in all patients, but mostly needed only one treatment to improve the condition completely. Acupuncture treatment with fire needles gives positive and very satisfying results for a very short time in the treatment of lateral epicondylitis, thereby relieving the pain and removing all the discomfort in the elbow.
\end{abstract}

Keywords: Acupuncture, Treatment, Traditional Chinese Medicine, Lateral Epicondylitis, Tennis Elbow

\section{Introduction}

Lateral epicondylitis i.e. tennis elbow usually occurs as overuse of the arm, forearm and the muscles in that area which result with pain in the elbow. A person doesn't have to play tennis to have this condition, it's just a common term used for inflammation of the extensor group of muscles in the arm (muscles and tendons in the external area of the elbow). The causes can be various, for example:

- Overuse of the arm,

- Repetitive, strong, monotonous or long movements,

- Repetitive extension of the wrist,

- Rotation of the forearm,

- Using the muscles very vigorously,

- Micro and major traumas and etc.

People who most suffer from this condition are those who regularly perform activities related with the movement of the hand when the palm is pointed down, jobs which involve repetitive heavy lifting, jobs with twisting or squeezing movements, gardening, cooking, moving house and etc. They usually complain of pain when lifting weight, doing pushups, intensive repetitive movements and etc. The common thing in all patients is the pain in the exterior side of the elbow. The pain usually does not appear immediately and it's bigger when shaking hands or squeezing some objects. Other symptoms are:

- Limited movements,

- Weakness,

- Stiffness,

- Fatigue and etc.

Sometimes a swelling can be present too. When these symptoms are present, the patients grip strength can be reduced, mainly when there's flexion in the elbow of 90 degrees.

The lateral epicondylitis appears in $1-3 \%$ of the population, between $40-50$ years of age and in $75 \%$ of the cases is affected the dominant arm. 
Tennis elbow is similar to the golfers elbow, except the pain is not on the outer side of the elbow, but on the inside (the flexor muscles and tendons are affected). The pain comes from the muscle extensor carpi radialis brevis, which function is wrist extension. [1] [2] [3]

Some authors refer to that tendinitis does not refer to tennis elbow (as is it usually thought of) but it needs to be seen as tendinopathy, because there is no such thing as inflamed tendons, instead there's been noticed a degeneration of the connective tissue. Computer work also can be a major cause for tennis elbow.

According to Western medicine, the treatment for tennis elbow is rest, wearing a brace, avoiding excessive use of the arm, occupational therapy, physical therapy, exercises, stretching, ultrasound, iontophoresis, hydrotherapy, icing, anti-inflammatory drugs or corticosteroid injections. Sometimes even surgery is proposed as an option.

In the Traditional Chinese Medicine the first choice for treatment is acupuncture, either with cold or hot needles. Some authors refer that acupuncture treatment should be done twice weekly in a period of four weeks to have the positive results. [3]

According to TCM the health issues appear as results of an internal imbalance of the body (the Qi, the blood, Yin, Yang). If the energy flow is blocked in some part of the body, it results with symptoms as pain. When treating some kind of sport injury, including tennis elbow, there will probably be some kind of form of blood and Qi stagnation, presented with pain and/or bruising and dampness involved in the affected meridians and collaterals, manifested as fluid retention. When treating a sport injury in an athlete, the practitioner must first examine the patient's general health condition because of excessive exercises and overstraining which may lead to Qi deficiency and weakness in the internal organs Liver, Stomach and Spleen. If the athlete's Qi energy is in deficit, he/she will be prone to invasion of the external pathogens like cold, wind and dampness. When the body is attacked of these external pathogens, the body's internal organs are affected and weakened. Because the Liver takes control over tendons, one of the theories is that lateral epicondylitis appears due to an insufficiency of Liver Yin. [4] [5] [6]

The aim of the acupuncture treatment is to stimulate the body to start the healing process, unblock all the energy pathways in the body, to release the pain and other symptoms, remove the discomfort, reduce the inflammation, improve the blood and Qi circulation, strengthen the tendons and etc. [7] [8]

\section{Material and Methods}

In this research are included 12 patients, 3 female and 9 male, on age from 24 to 70 . All the patients are diagnosed with lateral epicondylitis, all with symptoms of pain, discomfort, limited motion in the elbow and/or tingling. The patients information were retrieved from the list of patients treated in our clinic in a period of one year. Included patients were treated with fire needle acupuncture on trigger i.e. Ashi points. The numbers of the treatments were individual, but most of the patients needed only one treatment. The trigger points were found with palpation around the area of elbow and forearm on the most painful points. In the trigger points was inserted hot sterile tungsten steel needle with fast movement in and out with no retention, 5 times in each point. Treatments were done once a week in a clinic for Traditional Chinese Medicine and acupuncture in Skopje, by a doctor specialist in acupuncture, in a closed room on a temperature around $25^{\circ}$ with duration of the treatment of 3-5 minutes.

\section{Results and Discussion}

Before doing the acupuncture treatment, the patient is thorough examined, testing the entire arm, the shoulder, upper back and neck, where on palpation can be found a lot of trigger points. The posture of the patient should be evaluated and the range of motion too. We should notice all the symptoms and signs and the trigger activities that cause the tennis elbow symptoms. The cervical spine should be evaluated too, because the pain sometimes comes from the spine. When tennis elbow is present we might notice that activities which activate the trigger points and cause pain are those which include overload of the muscles and repetitive mechanical movements and stresses of the muscle. When the muscles and tendons are stressed over their own ability to repair themselves, then the inflammation is caused. When the muscles and tendons are continuously stressed and overused, they even hurt at rest. The trigger points are treated with direct needling with fire needle acupuncture. Needling the trigger points are especially helpful in patients with poor posture, who are working as desk workers, massage therapists or carpenters. If left untreated the condition can progress to calcification, bone spurs formations, periostitis and etc. [9] [10] Acupuncture treatment is effective because affected muscles and tendons are directly targeted. Therefore, the treatment helps the muscles to relax, normalize the blood flow and remove the blockages in the treated area. [11]

Because lateral epicondylitis is inflammation of a tendon, the treatment might take longer than in treating inflamed muscles, but with fire needle acupuncture treatment the results are even possible with one or two treatments. [5] In all our patients the treatment was effective with certain number of treatments. The symptoms that patients complained about before, after the treatments were gone. On Table 1. are shown the results from the number of treatments done.

Table 1. Number of treatment done.

\begin{tabular}{ll}
\hline Number of treatments & Number of patients \\
\hline 1 & 6 \\
2 & 2 \\
3 & 1 \\
4 & 1 \\
5 & 1 \\
$>5$ & 1 \\
\hline
\end{tabular}

Most of the patients (6) needed only one treatment to 
achieve positive results. Two patients needed two treatments and only one patient has done 3, 4, 5 and more than 5 treatments. In the research were included more male (9) than female (3) patients, which in our case is not of significance, however due to the statistics both genders are equally affected. According to the age, patients were on age from 24 to 70 , with most common age group of 30-40 and average age of 43, which matches with the statistics. [12] [13] The results from the age groups are shown on Table 2. Most of the patients (6) were on age from 30 to 40 . One patients in the group of 20-30 years, two in the age groups of 40-50 and 50-60 and only one patient in the group of older than 60 .

Table 2. Age groups.

\begin{tabular}{ll}
\hline Age groups & Number of patients \\
\hline $20-30$ & 1 \\
$30-40$ & 6 \\
$40-50$ & 2 \\
$50-60$ & 2 \\
$>60$ & 1 \\
\hline
\end{tabular}

According to the symptoms, most common symptom present in all patients was the pain in the lateral side of the elbow. Other symptoms were limited motion, tingling, tiredness, weakness and etc. According to the appearance of the condition some patients had the pain for over 5 years, some for 5-6 months and others for a shorter time. Some of the patients were chefs, professional athletes like handball and judo players or they got the injury from lifting heavy objects or excessive strain.

Acute or chronic pain, acupuncture can significantly help to improve the condition. In the terms of TCM tennis elbow can appear as a results of the external factors Wind and Cold, blood stasis or insufficient blood. The principle of the treatment is to regulate the Qi energy through the channels, free the flow of the energy, transform the stasis and quicken the blood. [14]

The treatment with fire needles is much different from the one with cold (normal) needles. With the fire needle acupuncture the harmful blood is removed physically and we get the results faster. Fire needle acupuncture helps the body naturally to repair the cells, dispel the stagnation, stimulate nerves, alleviate the joint pain and improve joint mobility, improve the microcirculation and the muscle stiffness, reduce inflammation, regulate body fluids, balances the Yin and Yang, dispel cold and wind and etc. [8] [15] [16] With the acupuncture treatment are stimulated the areas in the brain that are associated with pain, thereby the release of adenosine is increased which reduces the pain reactivity, nerves are stimulated to release endorphin, which is natural painkiller in the body, muscle stiffness is reduced and relaxation is promoted. [17]

In the treatment with fire needles are used trigger i.e. Ashi points, which are located on the most painful points found on palpation. In the area of the forearm and elbow can be found a lot of trigger points on palpation, even sometimes points go up to the neck, upper back, shoulders and in the area of lower back. One point is needled 5 times with quick insertion of the needle in and out.

According to the Chinese diagnosis, tennis elbow appears as a result of stagnation in the Qi and blood in the Li (Liver) channels. Pain often radiates from the elbow up and down along the Liver channels. The aim of the treatment is to move the Qi and blood into the Liver channels, so the pain can stop [10] The treatment is made with cold (normal) needles in the treatment usually are used the acupuncture points: Li10, Li11, Li12 and Lu5. Other authors suggest other points - Sp6, Sp9, Lr3, Lr8, St36 and other theories for tennis elbow occurrence - stagnation in the meridians of Triple energizer and Large intestine. [18] [19] The acupuncture points Li10, Li11, Li12 are local elbow ashi points and are used to move and stabilize the Qi and blood. The acupuncture point Tb10 is located on the elblow and it's also used to move the blood and Qi. The point St36 is distal point located on the leg and it is used do remove the blood and Qi stagnation. Li4 is an acupuncture point located on the hand and it is used to open and free the channels. Sp10 is a distal point located on the tight and it is used to move and balance the blood and Qi. [10] Also, there are many different techniques of needling and one very common technique used in the treatment of tennis elbow is eagle claw needling. [6]

Moxa can be added in the treatment when the weather is cold and damp so to alleviate blood stagnation and activate the Qi energy. Moxa can increase and warm the blood flow in the treated area and should not be used if there's swelling or redness [10] Some studies done for tennis elbow have come to a conclusion that acupuncture (including auricular acupuncture and electro-acupuncture) and moxibustion alone give much better results that some conventional treatments and sham acupuncture. Even better results are seen in combination of acupuncture and moxibustion (moxa cone placed on the acupuncture needle). [20]

With electro-acupuncture treatment are also seen good results in a study done for tennis elbow using the acupuncture points $\mathrm{St} 38$ and Gb34. Six treatments were done in two weeks with duration of the treatment of 20 minutes. The group if patients treated with electro-acupuncture showed better and more satisfying results than the group of patients treated with cold (normal) needle acupuncture. [10]

Other study proved that acupuncture treatment itself has a better result than steroid injections. 21 out of 34 patients who have done acupuncture treatment were totally pain free and in the controlled group with steroid injections out of 26 patients only 8 have improvement. [21]

In a systematic review of studies done for tennis elbow was concluded that acupuncture is a very effective treatment in short-term pain based on strong evidences. [22]

Active people who have tennis elbow should however continue to do smart and effective exercises and to avoid the future injuries they should do a progressive exercise program. [3]

\section{Conclusion}

Acupuncture treatment with fire needles gives positive and 
very satisfying results for a very short time in the treatment of lateral epicondylitis, thereby the pain is relieved, all discomfort in the joint is removed and the normal blood circulation in the affected area is enhanced.

\section{References}

[1] Петкова М.; Тениски и голф лакт; 2012 [www.doktori.mk].

[2] Harding M.; Tennis Elbow and Golfer's Elbow; 2015 [www.patient.info].

[3] Harrison L.; Tennis Elbow: What Is It, Do You Have It, And How Do You Treat It?; 2017 [www.breakingmuscle.com].

[4] Ingraham P.; Save Yourself from Tennis Elbow!; 2015 [www.painscience.com].

[5] Liu W., Gong C.; Acupuncture for Tennis Elbow; 2017 [www.tcmpage.com].

[6] Young K.; Sports Injuries and TCM; Journal of Chinese Medicine; Number 78; June 2005.

[7] Altshul S.; Ace Elbow Pain With Acupuncture; 2011 [www.prevention.com].

[8] Dupre E.; How to quickly treat tennis elbow with acupuncture? 2016 [www.wimi-fitness.com].

[9] Tapper S.; Tennis elbow / Lateral Epicondylitis; 2009 [www.suzannetapper.co.nz].

[10] Sitts C.; Acupuncture Treatment of Tennis Elbow; Research practicum; Summer 2015.

[11] Kilb J.; Acupuncture for Treating Tennis Elbow; 2012 [www.qiintegratedhealth.com].
[12] Miller J.; Tennis Elbow; 2017 [www.physioworks.com.au].

[13] Lerche O.; Pain in your forearm and difficulty opening doors? It could be a symptom of tennis elbow; 2016 [www.express.co.uk].

[14] Flaws B.; TheTuina Treatment of Tennis Elbow; Acupuncture Today; November, 2001, Vol. 02, Issue 11.

[15] Zhu J., Arsovska B., Kozovska K., Jovevska S.; Treatment of Knee Osteoarthritis with Fire Needle Acupuncture and Fire Cupping; Imperial Journal of Interdisciplinary Research (IJIR); Vol-2, Issue-11, 2016; ISSN: 2454-1362.

[16] Tcmwell.com; Acupuncture treatment of fire needle therapy; 2014 [www.tcmwell.com].

[17] Equilibre Acupuncture; Acupuncture for Tennis Elbow in Dublin 2; 2017 [www.equilibreacupuncture.ie].

[18] Binnendyk C.; What Are the Acupuncture Points for Tennis Elbow?; 2013 [www.livestrong.com].

[19] Clinical pearls; How Do You Treat Tennis Elbow in Your Practice?; Medical acupuncture; Volume 22, Number 2, 2010 Mary Ann Liebert, Inc. DOI: 10.1089=acu.2010.2013.

[20] Gadau M., Yeung W., Liu H. et al.; Acupuncture and moxibustion for lateral elbow pain: a systematic review of randomized controlled trials; BMC Complementary and Alternative Medicine; The official journal of the International Society for Complementary Medicine Research (ISCMR) 2014; $14: 136$.

[21] Brattberg G.; Acupuncture therapy for tennis elbow; Pain. 1983 Jul; 16 (3): 285-8.

[22] Trinh V. K.; Phillips D. S.; Ho E.; Domsma K.; Acupuncture for the alleviation of lateral epicondyle pain: a systematic review; Rheumatology (Oxford) (2004) 43 (9): 1085-1090. 